\title{
Differential Sensitivity of Brachial-Ankle Pulse Wave Velocity and Ankle-Brachial Index for Cardiovascular Risk Assessment in Elderly Chinese: A Cross-Sectional Study
}

\author{
Guoxiang $\mathrm{Fu}^{1}$, Yuan Zhong ${ }^{1}$, Fengfeng Pan ${ }^{1}$, Chanchan $\mathrm{Xu}^{2 *}$, (D) \\ ${ }^{1}$ Geriatrics Department, Shanghai Jiaotong University Affiliated Shanghai Sixth People's Hospital, Shanghai, China \\ ${ }^{2}$ Department of Internal Medicine, Shanghai Raffles Hospital, Shanghai, China
}

\section{ARTICLE INFO}

Article History

Received 21 August 2020

Accepted 15 December 2020

Keywords

Cardiovascular disease

brachial-ankle pulse wave velocity

ankle-brachial index

elderly Chinese

arterial stiffness

\begin{abstract}
Background: Brachial-Ankle Pulse Wave Velocity (baPWV) and Ankle-Brachial Index (ABI) are widely used in clinic for evaluating arterial stiffness and atherosclerosis separately. This study aimed to compare the sensitivity of baPWV and ABI for cardiovascular risk assessment in elderly Chinese.

Methods: BaPWV, ABI and Carotid Intima-Media Thickness (cIMT) were assessed in 169 elderly Chinese with the mean age of 85.7 years.

Results: Compared with normotensive participants, baPWV levels elevated in both limbs of hypertensive patients (2219 \pm $90 \mathrm{~cm} / \mathrm{s}$ vs. $1970 \pm 66 \mathrm{~cm} / \mathrm{s}, p=0.090 ; 2141 \pm 52 \mathrm{~cm} / \mathrm{s}$ vs. $1932 \pm 67 \mathrm{~cm} / \mathrm{s}, p=0.023$, separately). Compared with non-diabetes Mellitus (DM) participants, ABI levels declined in both limbs of DM patients $(0.96 \pm 0.02$ vs. $1.05 \pm 0.02, p=0.002$ and $0.99 \pm 0.03$ vs. $1.03 \pm 0.02, p=0.071$, separately). ABI of left limbs and ABI of right limbs were negatively correlated to cIMT ( $R=-0.136, p=0.090 ; R=-0.196, p=0.026$, separately) after adjusted by age, systolic pressure, diastolic pressure, body mass index, triglycerides, total cholesterol and low-density lipoprotein. The regression model for predicting Cardiovascular Disease (CVD) included the factors of gender, age, hypertension, baPWV of left limbs and baPWV of right limbs (OR $=6.701,95 \% \mathrm{CI}$ $0.911-49.310, p=0.062$; OR $=1.181,95 \%$ CI 1.051-1.327, $p=0.005$; OR $=0.214,95 \%$ CI $0.066-0.688, p=0.010 ;$ OR $=1.002$, $95 \%$ CI $1.000-1.003, p=0.065$; OR $=0.998,95 \%$ CI 0.996-0.999, $p=0.006$, separately).
\end{abstract}

Conclusion: In elderly Chinese, baPWV was elevated in hypertensive patients while ABI was lower in DM patients compared with healthy people. Compared with baPWV, ABI is better equivalent to cIMT. But for predicting the risk of CVD, baPWV is a better biomarker.

(C) 2021 The Authors. Publishing services by Atlantis Press International B.V. This is an open access article distributed under the CC BY-NC 4.0 license (http://creativecommons.org/licenses/by-nc/4.0/).

\section{INTRODUCTION}

Cardiovascular disease and cerebral vascular disease are the leading causes of mortality and morbidity worldwide [1-3]. Arterial stiffness has been recognized to be involved in the process of atherosclerosis $[4,5]$. Arterial stiffness is characterized by the loss of vessel elasticity and arterial compliance. The structural changes of the arterial wall are associated with aging, which are labeled by a decrease in the elastin content and collagen content of the arterial media [6].

Brachial-Ankle Pulse Wave Velocity (baPWV) and Ankle-Brachial Index $(\mathrm{ABI})$ are widely used in clinic for evaluating arterial stiffness and atherosclerosis separately $[7,8]$. But there is no guide for choice of method for evaluating cardiovascular risk in elderly population with different diseases, such as hypertension or Diabetes Mellitus (DM).

"Corresponding author. Email: chanchansh@163.com

Peer review under responsibility of the Association for Research into Arterial Structure and Physiology

Data availability statement: The authors confirm that the data supporting the findings of this study are available within the article [and/or] its supplementary materials.
Carotid Intima-Media Thickness (cIMT) is a marker of subclinical organ damage, which has been proved to predict Cardiovascular Disease (CVD) events in the general population, including with DM patients $[9,10]$.

Our study aimed to compare the sensitivity of baPWV and ABI for aging related cardiovascular risk assessment in elderly Chinese. The relation of baPWV and ABI to cIMT were analyzed in total subjects, hypertensive group and DM group. Moreover, potential factors for predicting CVD were also investigated in this study.

\section{MATERIALS AND METHODS}

\subsection{Subjects and Clinical Parameters}

Two hundred and two elderly individuals referred to Geriatrics department in Shanghai Sixth People's Hospital were evaluated. The inclusion criteria were: (1) aged above 65 years; (2) nonsmoker; (3) no alcoholic addiction. The exclusion criteria were: (1) tumor; (2) hepatic disease; (3) end-stage kidney disease (chronic 
kidney disease $\geq$ stage 4); (4) serious cardiovascular disease (acute myocardial infarction or New York Heart Association classification IV); (5) stroke within 3 months; (6) acute infection. A standard questionnaire was used to collect information, including age, gender, history of hypertension, history of DM, renal disease and use of antihypertensive or antidiabetic drugs or insulin. Resting blood pressure was measured using an automated oscillometric device (Terumo Elemano ES H5501, TERUMO Corporation, Hangzhou, China). Hypertension was defined by a blood pressure of 140/90 $\mathrm{mmHg}$ or more, or a history of taking antihypertensive medications. DM was defined as Fasting Plasma Glucose (FPG) equal or more than $7.0 \mathrm{mmol} / \mathrm{L}$, postprandial plasma glucose equal or more than $11.1 \mathrm{mmol} / \mathrm{L}$, or the use of hypoglycemic agents. Body weight and body height were measured by professionals, and Body Mass Index (BMI) was computed.

\subsection{Biological Parameters}

Blood samples were obtained when the participants were in a fasting state in the morning. Biological variants were tested in the clinical laboratory of Shanghai Sixth People's Hospital. Serum levels of FPG, Triglycerides (TG), Total Cholesterol (TC), Low-Density Lipoprotein cholesterol (LDL), High-Density Lipoprotein cholesterol (HDL) and creatinine were measured using an autoanalyzer (COBAS Integra 800, Roche Diagnostics, Basel, Switzerland). Hematocrit (HCT) determination was measured in blood samples using an automated blood analyzer (XE-5000, Sysmex, Kobe, Japan).

\subsection{Echocardiographic Examination}

Echocardiography was performed at Medical Ultrasound department of Shanghai Jiaotong University affiliated Shanghai Sixth People's Hospital. Echocardiographic assessment was performed with an ACUSON Sequoia ${ }^{\text {mi }} 512$ ultrasound system (Siemens Medical Solutions, Erlangen, Germany) equipped with an ultrabroad band frequency transducer (3-8 MHz). The Ejection Fraction (EF) was assessed using Simpson's biplane method.

\subsection{Brachial-Ankle Pulse Wave Velocity}

In this study, an Oscillometry-based device (BP-203RPE III; Colin Omron, Co, Ltd, Tokyo, Japan) was used to measure the baPWV. After rested for at least $10 \mathrm{~min}$, each subject was laid in the supine position with PWV cuffs wrapped around both ankles and upper arms. Bilateral PWV were recorded for analysis after the examination of both sides. All measurements were performed by trained clinician with the manufacturer's guide.

\subsection{Ankle-Brachial Index}

Ankle-brachial index was defined as the ratio of ankle and brachial Systolic Blood Pressure (SBP). Both ankle and brachial SBP were automatically and simultaneously measured by the validated device (VP-1000, Omron, Japan) on each side, the values of $\mathrm{ABI}$ could be read directly from the device. Bilateral
ABI were used for further analyses. Measurements were performed by the same staff member, who was trained with the manufacturer's guide.

\subsection{Carotid Artery Ultrasonography}

Carotid artery imaging was obtained by an experienced clinician using an ultrasound system (Acuson Sequoia 512, Siemens, Germany). The probe frequency was $7.0 \mathrm{MHz}$ and the axis resolution was $0.1 \mathrm{~mm}$. cIMT of Internal Carotid Artery (ICA), as the gap between the media-adventitia interface and lumen-intima interface, was measured $1.0 \mathrm{~cm}$ distal to both common carotid artery bifurcations. The measurement of IMT was carried out within a ICA region free of plaque.

\subsection{Statistical Analysis}

The unpaired Student's $t$-test, analysis of variance and analysis of covariance were used for analysis of continuous variables. Quantitative data were presented as the mean \pm standard error (S.E.), while categorical data are presented as numbers. Associations between variables were conducted using partial correlation analysis, multivariate linear stepwise regression analyses and logistic regression analysis. Two sided $p$-values $<0.05$ were considered significant and $p$-values $<0.1$ were considered slightly significant. The software SPSS 19.0 for Windows (SPSS Inc., IBM, USA) was used for statistical analysis.

\section{RESULTS}

\subsection{Clinical and Laboratory Characteristics}

Of the original 202 participants, 12 dropped out of the study and 21 were excluded because of various diseases, leaving 169 participants who were included in this cross-sectional study. Of the 169 participants, 119 were diagnosed with hypertension $(70.4 \%)$ and 62 were diagnosed with DM (36.7\%). There were 150 male participants $(88.2 \%)$. The mean age was $85.7 \pm 0.4$ years, the mean Systolic Blood Pressure (SBP) was $124 \mathrm{mmHg}$, the mean Diastolic Blood Pressure (DBP) was $70 \mathrm{mmHg}$ and the mean BMI was $23.8 \pm 0.3 \mathrm{~kg} / \mathrm{m}^{2}$. Laboratory parameters, PWV, ABI, and ultrasound parameters are reported in Table 1.

\subsection{Comparison of Clinical Characteristics and baPWV between Hypertension Group and Normotension Group}

As shown in Table 2, we observed higher TG concentrations and lower TC concentrations in hypertension group than in normotension group. The levels of age, SBP, DBP, BMI, FPG, LDL, HDL, HCT, creatinine, EF and IMT were not significantly different between hypertension group and normotension group. Compared with normotensive participants, baPWV levels of hypertensive patients were slightly higher in left limbs $(2219 \pm 90 \mathrm{~cm} / \mathrm{s}$ vs. 
$1970 \pm 66 \mathrm{~cm} / \mathrm{s}, p=0.090)$ and significantly higher in right limbs $(2141 \pm 52 \mathrm{~cm} / \mathrm{s}$ vs. $1932 \pm 67 \mathrm{~cm} / \mathrm{s}, p=0.023)$ separately (Table 2).

\subsection{Comparison of ABI between Hypertension Group and Normotension Group}

Ankle-brachial index levels of hypertension group were compared with those of normotension group. The ABI levels of right limbs in hypertensive patients were slightly lower than those of normotensive participants $(1.00 \pm 0.02$ vs. $1.06 \pm 0.02, p=0.049)$. But no difference of ABI levels between hypertensive group and normotensive group was observed in left limbs (Table 2).

Table 1 Clinical characteristics of total subjects

\begin{tabular}{lcc}
\hline Clinical characteristics & Mean value & SE \\
\hline FPG $(\mathrm{mmol} / \mathrm{L})$ & 5.64 & 0.13 \\
TG $(\mathrm{mmol} / \mathrm{L})$ & 1.89 & 0.12 \\
TC $(\mathrm{mmol} / \mathrm{L})$ & 3.36 & 0.12 \\
LDL $(\mathrm{mmol} / \mathrm{L})$ & 2.16 & 0.06 \\
HDL $(\mathrm{mmol} / \mathrm{L})$ & 1.09 & 0.03 \\
HCT $(\%)$ & 38.4 & 0.5 \\
Creatinine ( $\mu \mathrm{mol} / \mathrm{L})$ & 89.1 & 3.0 \\
L-PWV $(\mathrm{cm} / \mathrm{s})$ & 2145 & 67 \\
R-PWV (cm/s) & 2079 & 42 \\
L-ABI & 1.01 & 0.01 \\
R-ABI & 1.02 & 0.01 \\
EF $(\%)$ & 63 & 0.69 \\
IMT $(\mathrm{mm})$ & 0.87 & 0.01 \\
\hline
\end{tabular}

FPG, fasting plasma glucose; TG, triglycerides, TC, total cholesterol; LDL, low-density lipoprotein; HDL, high-density lipoprotein; HCT, red blood cell specific volume; baPWV, brachial-ankle pulse wave velocity; L-PWV, baPWV in left limbs; R-PWV, baPWV in right limbs; ABI, ankle brachial index; L-ABI, ABI in left limbs; R-ABI, ABI in right limbs; EF, ejection fraction; cIMT, carotid intima media thickness.

Table 2 Clinical characteristics between hypertensive patients and normotensive participants

\begin{tabular}{lccc}
\hline Clinical parameters & Hypertension & Normotension & $p$-value \\
\hline$n$ & 119 & 50 & - \\
Age (years) & $86.1 \pm 0.4$ & $84.8 \pm 0.9$ & 0.156 \\
SBP $(\mathrm{mmHg})$ & $124 \pm 1$ & $125 \pm 1$ & 0.367 \\
DBP $(\mathrm{mmHg})$ & $70 \pm 1$ & $70 \pm 1$ & 0.873 \\
BMI & $23.7 \pm 0.3$ & $24.0 \pm 0.5$ & 0.670 \\
FPG $(\mathrm{mmol} / \mathrm{L})$ & $5.76 \pm 0.18$ & $5.40 \pm 0.05$ & 0.215 \\
TG $(\mathrm{mmol} / \mathrm{L})$ & $2.09 \pm 0.16^{*}$ & $1.48 \pm 0.17^{*}$ & 0.018 \\
TC $(\mathrm{mmol} / \mathrm{L})$ & $3.09 \pm 0.16^{*}$ & $3.92 \pm 0.18^{*}$ & 0.002 \\
LDL $(\mathrm{mmol} / \mathrm{L})$ & $2.11 \pm 0.08$ & $2.30 \pm 0.11$ & 0.194 \\
HDL $(\mathrm{mmol} / \mathrm{L})$ & $1.07 \pm 0.05$ & $1.10 \pm 0.06$ & 0.760 \\
HCT $(\%)$ & $38.0 \pm 0.6$ & $39.3 \pm 0.7$ & 0.607 \\
Creatinine $(\mu \mathrm{mol} / \mathrm{L})$ & $92.3 \pm 3.8$ & $83.1 \pm 4.5$ & 0.141 \\
L-PWV $(\mathrm{cm} / \mathrm{s})$ & $2219 \pm 90^{*}$ & $1970 \pm 66^{*}$ & 0.090 \\
R-PWV (cm/s) & $2141 \pm 52^{*}$ & $1932 \pm 67^{*}$ & 0.023 \\
L-ABI & $1.00 \pm 0.02$ & $1.03 \pm 0.02$ & 0.313 \\
R-ABI & $1.00 \pm 0.02^{*}$ & $1.06 \pm 0.02^{*}$ & 0.049 \\
EF $(\%)$ & $63.4 \pm 0.6$ & $62.3 \pm 1.9$ & 0.534 \\
IMT $(\mathrm{mm})$ & $0.86 \pm 0.01$ & $0.88 \pm 0.03$ & 0.01 \\
\hline
\end{tabular}

${ }^{*} p<0.05 ;{ }^{*} p<0.1$.

\subsection{Comparison of Clinical Characteristics and baPWV between DM Group and Non-DM Group}

Fasting plasma glucose concentrations of DM patients were higher, while TC concentrations of DM patients were lower than those of non-DM participants. The levels of age, SBP, DBP, BMI, FPG, LDL, HDL, HCT, creatinine, EF and IMT were not significantly different between DM group and non-DM group. No difference was observed between PWV levels of DM patients and non-DM participants either in left limbs or in right limbs. TG concentrations were slightly higher and EF levels were slightly lower in DM patients compared to non-DM participants (Table 3 ).

\subsection{Comparison of ABI between DM Group and Non-DM Group}

Compared with non-DM participants, ABI levels of DM patients were significantly lower in left limbs $(0.96 \pm 0.02$ v.s. $1.05 \pm 0.02$, $p=0.002)$. In right limbs, the ABI levels of DM group were slightly lower than non-DM group $(0.99 \pm 0.03$ vs. $1.03 \pm 0.02, p=0.071)$ (Table 3).

\subsection{Correlation between baPWV, $\mathrm{ABI}$ and CIMT}

Both ABI of Left Limbs (L-ABI) and ABI of Right Limbs (R-ABI) were negatively correlated to cIMT in partial correlation analysis after adjusted by age, SBP, DBP, BMI, TC, TG and LDL ( $R=$ $-0.136, p=0.090 ; R=-0.196, p=0.026$, separately). However, neither baPWV of Left Limbs (L-PWV) nor baPWV of Right Limbs (R-PWV) was correlated to cIMT (Table 4).

Table 3 Clinical characteristics between DM patients and non-DM participants

\begin{tabular}{lccc}
\hline Clinical parameters & Diabetes mellitus & $\begin{array}{c}\text { Non-Diabetes } \\
\text { mellitus }\end{array}$ & p-value \\
\hline$n$ & 62 & 107 & - \\
Age (years) & $85.9 \pm 0.6$ & $85.5 \pm 0.5$ & 0.638 \\
SBP (mmHg) & $124 \pm 1$ & $124 \pm 1$ & 0.577 \\
DBP (mmHg) & $70 \pm 1$ & $70 \pm 1$ & 0.494 \\
BMI & $23.7 \pm 0.5$ & $23.8 \pm 0.3$ & 0.818 \\
FPG (mmol/L) & $6.69 \pm 0.34^{*}$ & $5.18 \pm 0.05^{*}$ & 0.000 \\
TG (mmol/L) & $2.21 \pm 0.23^{*}$ & $1.73 \pm 0.13^{*}$ & 0.062 \\
TC (mmol/L) & $2.90 \pm 0.22^{*}$ & $3.59 \pm 0.15^{*}$ & 0.008 \\
LDL (mmol/L) & $2.13 \pm 0.12$ & $2.20 \pm 0.08$ & 0.624 \\
HDL (mmol/L) & $1.04 \pm 0.07$ & $1.11 \pm 0.04$ & 0.377 \\
HCT & $37.0 \pm 0.8$ & $39.1 \pm 0.5$ & 0.607 \\
Creatinine ( $\mu$ mol/L) & $94.6 \pm 5.6$ & $86.2 \pm 3.4$ & 0.180 \\
L-PWV & $2141 \pm 90$ & $2148 \pm 92$ & 0.964 \\
R-PWV & $2126 \pm 71$ & $2053 \pm 52$ & 0.405 \\
L-ABI & $0.96 \pm 0.02^{*}$ & $1.05 \pm 0.02^{*}$ & 0.002 \\
R-ABI & $0.99 \pm 0.03^{*}$ & $1.03 \pm 0.02^{*}$ & 0.076 \\
EF $(\%)$ & $61.7 \pm 1.4^{*}$ & $64.2 \pm 0.6^{*}$ & 0.071 \\
cIMT (mm) & $0.86 \pm 0.02$ & $0.88 \pm 0.02$ & 0.636 \\
\hline
\end{tabular}

${ }^{*} p<0.05 ; " p<0.1$. 
Stepwise regression analysis of baPWV and ABI to cIMT was conducted in hypertensive group and DM group separately. In hypertensive patients, neither baPWV nor ABI was included in the model for cIMT. In DM patients, R-ABI was included in the model for predicting $\operatorname{cIMT}(\beta=-0.316,95 \% \mathrm{CI}-0.562$ to $-0.071, p=0.012)$.

\subsection{Sensitivity of baPWV and ABI for Predicting CVD}

As for early prediction of disease caused by arterial stiffness, logistic regression analysis of CVD with the factors of age, gender, SBP, DBP, BMI, DM, hypertension, L-PWV, R-PWV, L-ABI, R-ABI, cIMT, TC, TG and LDL. The model for predicting CVD included the factors of gender, age, hypertension, L-PWV and R-PWV $(\mathrm{OR}=6.701,95 \% \mathrm{CI} 0.911-49.310, p=0.062 ; \mathrm{OR}=1.181,95 \% \mathrm{CI}$ $1.051-1.327, p=0.005 ; \mathrm{OR}=0.214,95 \%$ CI $0.066-0.688, p=0.010$; $\mathrm{OR}=1.002,95 \%$ CI $1.000-1.003, p=0.065$; OR $=0.998,95 \% \mathrm{CI}$ $0.996-0.999, p=0.006$, separately) (Table 5).

\section{DISCUSSION}

Population aging leads to more patients suffered from diseases related to arterial stiffness and atherosclerosis, such as cardiovascular disease, cerebral vascular disease and diabetes nephropathy. Not only high plasma glucose, lipid and high blood pressure, but also aging itself can cause arterial stiffness [11]. Our study included subjects at an average age of 85 years old, which represented elderly Chinese population. To identify better method of evaluating cardiovascular risks in elderly hypertensive patients, baPWV levels of hypertension group were compared with those of normotension group.

We found baPWV levels were elevated in both limbs of hypertensive patients compared with non-hypertensive subjects. However, only ABI of right limb was slightly lower in hypertensive patients.

Table 4 Partial correlation analysis between baPWV, ABI and cIMT after adjusted by age, SBP, DBP, BMI, TC, TG and LDL

\begin{tabular}{lcc}
\hline Variants & $\boldsymbol{r}$ & $\boldsymbol{p}$-value \\
\hline L-PWV & 0.091 & 0.184 \\
R-PWV & 0.045 & 0.331 \\
L-ABI & $-0.136^{\#}$ & 0.090 \\
R-ABI & $-0.196^{*}$ & 0.026 \\
\hline
\end{tabular}

$r$, Partial correlation coefficient; ${ }^{*} p<0.1 ;{ }^{*} p<0.05$.

Table 5 Logistic regression analysis on risk factors for predicting cardiovascular disease (CVD)

\begin{tabular}{lccc}
\hline Factors & OR & $\mathbf{9 5 \%} \mathbf{C I}$ & $\boldsymbol{p}$-value \\
\hline Gender & $6.701^{\#}$ & $0.911-49.310$ & 0.062 \\
Age & $1.181^{*}$ & $1.051-1.327$ & 0.005 \\
Hypertension & $0.214^{*}$ & $0.066-0.688$ & 0.010 \\
L-PWV & $1.002^{\#}$ & $1.000-1.003$ & 0.065 \\
R-PWV & $0.998^{*}$ & $0.996-0.999$ & 0.006 \\
\hline
\end{tabular}

Logistic regression analysis of CVD with the factors of age, gender, SBP, DBP, BMI, DM, hypertension, L-PWV, R-PWV, L-ABI, R-ABI, cIMT, TC, TG and LDL. Data are odds ratio (OR) and $95 \%$ confidence interval $(95 \% \mathrm{CI}) .{ }^{*} p<0.1 ;{ }^{*} p<0.05$.
We deduce baPWV might be better method for evaluating arterial stiffness in elderly hypertensive patients. Munakata et al. [12] reported high baPWV was associated with a significantly poorer outcome in hypertensive Japanese adult at the average age of 60 years. Our study implied this rule might be also applicable in even older hypertensive Chinese.

To identify better method of evaluating cardiovascular risks in elderly DM patients, we compared PWV and ABI levels of DM group with those of non-DM group. ABI levels of DM patients were lower in both limbs compared with non-DM participants. But no significant difference of PWV was observed between DM patients and non-DM participants. ABI might be better method for evaluating DM related vascular disease. Our finding is consistent with the recommendation of $\mathrm{ABI}$ as non-invasive tool to diagnose peripheral artery diseases in diabetes patients $[13,14]$.

We found both L-ABI and R-ABI, but not baPWV, were significantly correlated to cIMT in the total study subjects. Furthermore, R-ABI was included in the model for predicting cIMT in DM patients. Compared with baPWV, better equivalence of ABI to cIMT was revealed in our study. CIMT has been reported to be a biomaker for CVD $[15,16]$. Combined with the result of our study, both ABI and cIMT might be associated with macro-vascular damage.

To investigate the real roles of baPWV and $\mathrm{ABI}$ as risk factors for CVD in the total subjects with and without hypertension or DM, logistic regression analysis were conducted. L-PWV and R-PWV, together with gender, age and hypertension, were included in the regression model for CVD. In previous study, arotic PWV was found to be associated with higher cardiovascular mortality, coronary heart disease and stroke among generally healthy older adults at mean age of 73.4 years [17]. baPWV has been reported to enhance the efficacy of prediction for the risk of development of CVD over that of the Framingham risk score, which is based on the traditional cardiovascular risk factors $[18,19]$. Our study confirmed the efficacy of baPWV in cardiovascular risk evaluation in elderly Chinese over 80 years old.

\section{CONCLUSION}

In elderly Chinese over 80 years' old, baPWV was elevated in hypertensive patients while ABI was lower in DM patients compared with healthy people. Compared with baPWV, ABI is better equivalent to cIMT. But for predicting risk of CVD, baPWV is a better biomarker in elderly Chinese.

\section{CONFLICTS OF INTEREST}

The authors declare they have no conflicts of interest.

\section{AUTHORS' CONTRIBUTION}

$\mathrm{CX}, \mathrm{GF}$ and $\mathrm{YZ}$ were involved in the conception of the work. CX, $\mathrm{GF}$ and FP were involved in data curation. CX and GF were involved in statistical analysis. CX was involved in the final approval of the version to be published. CX, GF, YZ and FP were involved in the whole process of writing, reviewing and editing. 


\section{FUNDING}

This study was supported by Shanghai Shen Kang Hospital Development Center Clinical Research Training Project (Grant No. SHDC12017X21). The funding body had no role in the study design; collection, analysis, and interpretation of data; writing of the report; or decision to submit the article for publication.

\section{ACKNOWLEDGMENTS}

We gratefully acknowledge the invaluable assistance offered by the physicians in the Geriatrics Department of Shanghai Jiaotong University affiliated Shanghai Sixth people's Hospital.

\section{ABBREVIATIONS}

BaPWV, brachial-ankle pulse wave velocity; ABI, ankle-brachial index; DM, diabetes mellitus; FPG, fasting plasma glucose; BMI, body mass index; TG, triglycerides; TC, total cholesterol; LDL, low-density lipoprotein; HDL, highdensity lipoprotein; HCT, hematocrit determination; SBP, systolic blood pressure; DBP, diastolic blood pressure; EF, ejection fraction; cIMT, carotid intima-media thickness; L-PWV, baPWV of left limbs; R-PWV, baPWV of right limbs; L-ABI, ABI of left limbs; R-ABI, ABI of right limbs.

\section{ETHICS APPROVAL AND CONSENT TO PARTICIPATE}

The protocol of the study was approved by the ethics committee of Shanghai Jiaotong University Affiliated Sixth People's Hospital (Shanghai, China). Each participate gave written informed consent to the procedure.

\section{REFERENCES}

[1] Naghavi M, Abajobir AA, Abbafati C, Abbas KM, Abd-Allah F, Abera SF, et al. Global, regional, and national age-sex specific mortality for 264 causes of death, 1980-2016: a systematic analysis for the Global Burden of Disease Study 2016. Lancet 2017;390:1151-210.

[2] Saji N, Toba K, Sakurai T. Cerebral small vessel disease and arterial stiffness: tsunami effect in the brain? Pulse 2015;3:182-9.

[3] Tuttolomondo A, Pecoraro R, Di Raimondo D, Di Sciacca R, Canino B, Arnao V, et al. Immune-inflammatory markers and arterial stiffness indexes in subjects with acute ischemic stroke with and without metabolic syndrome. Diabetol Metab Syndr 2014;6:28.

[4] Boutouyrie P, Tropeano AI, Asmar R, Gautier I, Benetos A, Lacolley $\mathrm{P}$, et al. Aortic stiffness is an independent predictor of primary coronary events in hypertensive patients: a longitudinal study. Hypertension 2002;39:10-15.

[5] Arnett DK, Boland LL, Evans GW, Riley W, Barnes R, Tyroler HA, et al. Hypertension and arterial stiffness: the Atherosclerosis Risk in Communities Study. ARIC Investigators. Am J Hypertens 2000;13:317-23.
[6] Nilsson PM, Boutouyrie P, Cunha P, Kotsis V, Narkiewicz K, Parati $G$, et al. Early vascular ageing in translation: from laboratory investigations to clinical applications in cardiovascular prevention. J Hypertens 2013;31:1517-26.

[7] Ohkuma T, Tomiyama H, Ninomiya T, Kario K, Hoshide S, Kita Y, et al. Proposed cutoff value of brachial-ankle pulse wave velocity for the management of hypertension. Circ J 2017;81:1540-2.

[8] Guirguis-Blake JM, Evans CV, Redmond N, Lin JS. Screening for peripheral artery disease using the ankle-brachial index updated evidence report and systematic review for the US preventive services task force. JAMA 2018;320:184-96.

[9] Lorenz MW, Polak JF, Kavousi M, Mathiesen EB, Völzke H, Tuomainen TP, et al. Carotid intima-media thickness progression to predict cardiovascular events in the general population (the PROG-IMT collaborative project): a meta-analysis of individual participant data. Lancet 2012;379:2053-62.

[10] Lorenz MW, Price JF, Robertson C, Bots ML, Polak JF, Poppert $\mathrm{H}$, et al. Carotid intima-media thickness progression and risk of vascular events in people with diabetes: results from the PROGIMT collaboration. Diabetes Care 2015;38:1921-9.

[11] O’Rourke MF, Safar ME, Dzau V. The cardiovascular continuum extended: aging effects on the aorta and microvasculature. Vasc Med 2010;15:461-8.

[12] Munakata M, Konno S, Miura Y, Yoshinaga K, J-TOPP Study Group. Prognostic significance of the brachial-ankle pulse wave velocity in patients with essential hypertension: final results of the J-TOPP study. Hypertens Res 2012;35:839-42.

[13] Nativel M, Potier L, Alexandre L, Baillet-Blanco L, Ducasse E, Velho G, et al. Lower extremity arterial disease in patients with diabetes: a contemporary narrative review. Cardiovasc Diabetol 2018;17:138.

[14] Norman PE, Davis WA, Bruce DG, Davis TM. Peripheral arterial disease and risk of cardiac death in type 2 diabetes: the Fremantle Diabetes Study. Diabetes Care 2006;29:575-80.

[15] Urbina EM, Williams RV, Alpert BS, Collins RT, Daniels SR, Hayman L, et al. Noninvasive assessment of subclinical atherosclerosis in children and adolescents: recommendations for standard assessment for clinical research: a scientific statement from the American Heart Association. Hypertension 2009;54:919-50.

[16] Eikendal AL, Groenewegen KA, Bots ML, Peters SA, Uiterwaal CS, den Ruijter HM. Relation between adolescent cardiovascular risk factors and carotid intima-media echogenicity in healthy young adults: the atherosclerosis risk in young adults (ARYA) study. J Am Heart Assoc 2016;5:e002941.

[17] Sutton-Tyrrell K, Najjar SS, Boudreau RM, Venkitachalam L, Kupelian V, Simonsick EM, et al. Elevated aortic pulse wave velocity, a marker of arterial stiffness, predicts cardiovascular events in well-functioning older adults. Circulation 2005; 111:3384-90.

[18] Ohkuma T, Ninomiya T, Tomiyama H, Kario K, Hoshide S, Kita $\mathrm{Y}$, et al. Brachial-ankle pulse wave velocity and the risk prediction of cardiovascular disease: an individual participant data meta-analysis. Hypertension 2017;69:1045-52.

[19] Ninomiya T, Kojima I, Doi Y, Fukuhara M, Hirakawa Y, Hata J, et al. Brachial-ankle pulse wave velocity predicts the development of cardiovascular disease in a general Japanese population: the Hisayama Study. J Hypertens 2013;31:477-83. 